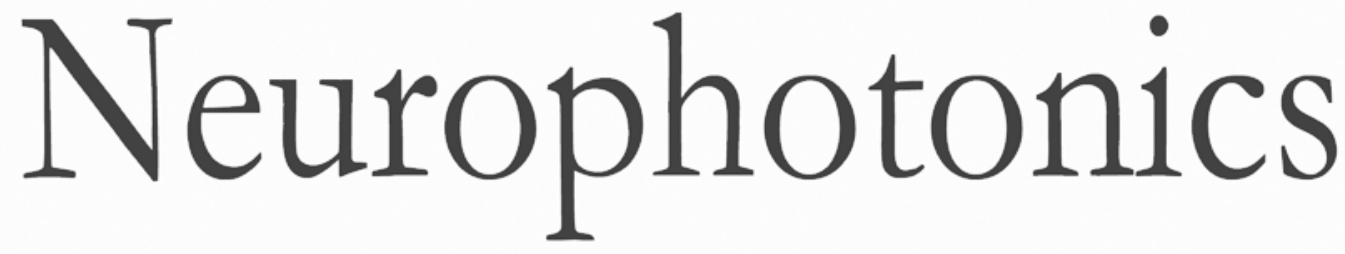

\title{
Interpreting in vivo calcium signals from neuronal cell bodies, axons, and dendrites: a review
}

Farhan Ali

Alex C. Kwan 


\title{
Interpreting in vivo calcium signals from neuronal cell bodies, axons, and dendrites: a review
}

\author{
Farhan Ali and Alex C. Kwan ${ }^{\mathrm{a}, \mathrm{b}, *}$ \\ aYale University, Department of Psychiatry, School of Medicine, New Haven, Connecticut, United States \\ bYale University, Department of Neuroscience, School of Medicine, New Haven, Connecticut, United States
}

\begin{abstract}
Calcium imaging is emerging as a popular technique in neuroscience. A major reason is that intracellular calcium transients are reflections of electrical events in neurons. For example, calcium influx in the soma and axonal boutons accompanies spiking activity, whereas elevations in dendrites and dendritic spines are associated with synaptic inputs and local regenerative events. However, calcium transients have complex spatiotemporal dynamics, and since most optical methods visualize only one of the somatic, axonal, and dendritic compartments, a straightforward inference of the underlying electrical event is typically challenging. We highlight experiments that have directly calibrated in vivo calcium signals recorded using fluorescent indicators against electrophysiological events. We address commonly asked questions such as: Can calcium imaging be used to characterize neurons with high firing rates? Can the fluorescent signal report a decrease in spiking activity? What is the evidence that calcium transients in subcellular compartments correspond to distinct presynaptic axonal and postsynaptic dendritic events? By reviewing the empirical evidence and limitations, we suggest that, despite some caveats, calcium imaging is a versatile method to characterize a variety of neuronal events in vivo. (C) The Authors. Published by SPIE under a Creative Commons Attribution 4.0 Unported License. Distribution or reproduction of this work in whole or in part requires full attribution of the original publication, including its DOI. [DOI: 10.1117/1.NPh.7.1.011402]
\end{abstract}

Keywords: calcium imaging; two-photon microscopy; fluorescence; neuron; calibration.

Paper 19027SSRR received Mar. 20, 2019; accepted for publication Jul. 2, 2019; published online Jul. $30,2019$.

\section{Introduction}

Calcium imaging refers to optical methods for measuring the concentration of calcium ions in cells. In neuroscience, there has been an explosion in the number of studies that employ calcium imaging. For example, the method has been applied to awake animals to study, at the subcellular level, the role of dendritic calcium signals during perceptual performance; ${ }^{1}$ at the singleneuron level, learning-related neural activity across the course of motor skill acquisition; ${ }^{2}$ at the local-circuit level, dynamics of frontal cortical ensembles during decision-making; ${ }^{3}$ and, at the network level, interactions between brain regions that underlie neurodevelopment. ${ }^{4}$ Calcium imaging is also the primary method behind a recent large-scale effort to survey neural responses in the mouse visual cortex. ${ }^{5}$

The rising popularity of calcium imaging is due in part to technical advances. The development of genetically encoded calcium indicators (GECIs) - fluorescent proteins that sense calcium and report via a change in emission amplitude or spectrum-enabled measurements with high signal-to-noise ratios. ${ }^{6}$ Further work in protein engineering led to GECI variants with attractive properties such as a redshifted emission spectrum. ${ }^{7}$ These GECIs can be introduced not only using viruses but are also available in a variety of transgenic animals. ${ }^{8}$ Moreover, there are mature optical techniques for subcellular-resolution imaging in the intact brain based on two-photon-excited fluorescence. ${ }^{9}$ New microscope designs additionally enable imaging deep in scattering tissues ${ }^{10}$ and across a wide field of view. ${ }^{11}$ Collectively, these advances have led to the widespread adoption of calcium imaging as a tool for neuroscience research.

*Address all correspondence to Alex C. Kwan, E-mail: alex.kwan@yale.edu
A major reason for measuring calcium is that the influx of calcium ions is associated with electrical events, such as synaptic activation and dendritic spikes, that are difficult to measure with other methods. The inference is possible because we have accrued knowledge of the biophysical mechanisms that regulate the entry and life cycle of calcium ions in neuronal compartments. The inference is made stronger by computational models that describe calcium measurements in cellular compartments, which allows for a better dissociation between the desired goal to measure free calcium ions and the side effect of buffering by the calcium indicators. ${ }^{12,13}$ These topics have been discussed extensively in other review articles that have focused on the utility of calcium imaging as a tool in neuroscience, ${ }^{14-16}$ the mechanisms that regulate calcium in neurons, ${ }^{17-20}$ the quantitative aspects of sensing calcium with fluorophores, ${ }^{21-23}$ and procedures for analyzing calcium imaging data. ${ }^{24}$

This article is intended to be a primer for interpreting calcium signals recorded from neurons with fluorescent indicators in vivo. Namely, if we see a fluorescent transient in the cell body, axon, or dendrite, what does the signal mean? Are the spatiotemporal dynamics consistent with a cellular event? Moreover, if the signal seems physiological, what can we say about the electrical event that gives rise to the calcium transient, and what in vivo calibration data would support the conclusion? Calibration is particularly relevant because in vivo neural dynamics are affected by background activity, neuromodulation, and GABAergic inputs, such that calcium signals could be markedly different in in vivo versus in vitro conditions. With these questions in mind, the main text is divided into sections based on various neural correlates commonly assigned to in vivo calcium signals. Within each section, we briefly introduce the biophysical basis, and then focus on experiments that have directly calibrated in vivo calcium signals against 
electrophysiological events. We highlight empirical details that are important for proper interpretation of calcium imaging data. We end each section by discussing limitations. We primarily use neocortical neurons as examples because many types of in vivo calibration data are available.

\section{Somatic Calcium Signals: as a Proxy for Spiking Activity}

Somatic calcium signals have been used to infer spiking activity in neurons. By imaging an ensemble of neurons at singlecell resolution, one could delineate task-related neural activity during perceptual or cognitive behavior, ${ }^{25}$ map the spatial organization of visually evoked neural responses, ${ }^{26}$ and track modifications to neural activity across learning. ${ }^{27}$

An action potential causes calcium influx in the neuronal cell body. For example, in layer 5 pyramidal neurons in the neocortex, calcium enters the soma through voltage-dependent calcium channels, including L-type channels that open in response to a large depolarization $(\sim 20 \mathrm{mV})$, as well as $\mathrm{N}$-, and P/Q-type channels. ${ }^{28}$ The resting calcium concentration at the soma is $\sim 50 \mathrm{nM}$, and the increase due to a single action potential is estimated to be $\sim 40 \mathrm{nM}^{29}$ This number may underestimate the actual amplitude, because even a moderate amount of fluorescent indicators would buffer and prolong the life cycle of calcium ions in a cell. ${ }^{13,30}$ Measurements in the nearby proximal apical dendrite (30 to $50 \mu \mathrm{m}$ from soma) suggest a rapid rise time ( $<2$ ms to $90 \%$ of the peak amplitude; the measurement was limited by the kinetics of fluorescent indicators), and a decay time constant of $\sim 70 \mathrm{~ms} .{ }^{13}$ At the cell body, because of larger cytosolic volume, the decay time constant should be larger than the value at the proximal apical dendrite, and one study estimates a factor of $\sim 150 \%$. $^{29}$ Therefore, although the action potential is a millisecond-scale event, the somatic calcium elevation has a duration that is longer by about two orders of magnitude.

Many studies have imaged calcium signals while recording spiking activity from the cell body in vivo to show a direct relationship between somatic calcium transients and action potentials. ${ }^{6,31-33}$ In one example, ${ }^{6}$ putative excitatory somata were targeted for simultaneous two-photon calcium imaging using the fluorescent indicator GCaMP6s or GCaMP6f and juxtacellular recording in the neocortex of anesthetized mice [Fig. 1(a)]. Fluorescence rose with each action potential [Fig. 1(b)]. It should be emphasized that the signal reported by a fluorescent indicator is a convolution of the calcium transients and the indicator response. The choice of indicator matters, as evident by the different fluorescence profiles in response to a single spike for the two GECIs [Fig. 1(c)]. In this case, GCaMP6s has slower single-spike kinetics (time to peak of $179 \mathrm{~ms}$ and decay time of $550 \mathrm{~ms}$ ) compared to GCaMP6f (45 and $142 \mathrm{~ms}$, respectively). ${ }^{6}$

Furthermore, in the intervening periods between action potentials, the fluorescence was mostly unchanged [Fig. 1(a)], suggesting that calcium elevations occurred only from suprathreshold depolarizations. This is consistent with the biophysical properties of the high-threshold calcium channels in the cell body. In practice, for cortical pyramidal neurons, it is possible to find a weak correlation between the subthreshold fluctuations in membrane potentials and the somatic calcium signals recorded in vivo. However, the source of that weak correlation probably does not come from the imaged cell, but rather originates from contamination of fluorescence signals from the surrounding neuropil, which would reflect the local network activity and correlate with membrane potentials of the imaged cell [for an example, see Supplementary Figs. 1(e) and 1(f) in Ref. 32].

When action potentials are sparse relative to the imaging frame rate, a "kernel" can be estimated to describe the fluorescence response to a single action potential [Fig. 1(c)]. Deconvolution of this kernel from the time-lapse fluorescent signal would yield spike times. ${ }^{34,35}$ Having the kernel also enables other types of analyses. For example, spike-field coherence has been used to determine phase synchronization between neuronal firing and local oscillatory activity. ${ }^{36}$ Analogously, from calcium imaging and field recordings, a fluorescence-field coherence could be determined. In one study that imaged spinal neurons while recording from motor nerves, the coherence allows for quantification of the locomotion phase during which the neurons contribute to motor pattern generation. ${ }^{37}$ The kernel is required to account for the kinetics of the indicator to ensure the phase is accurate. (a)

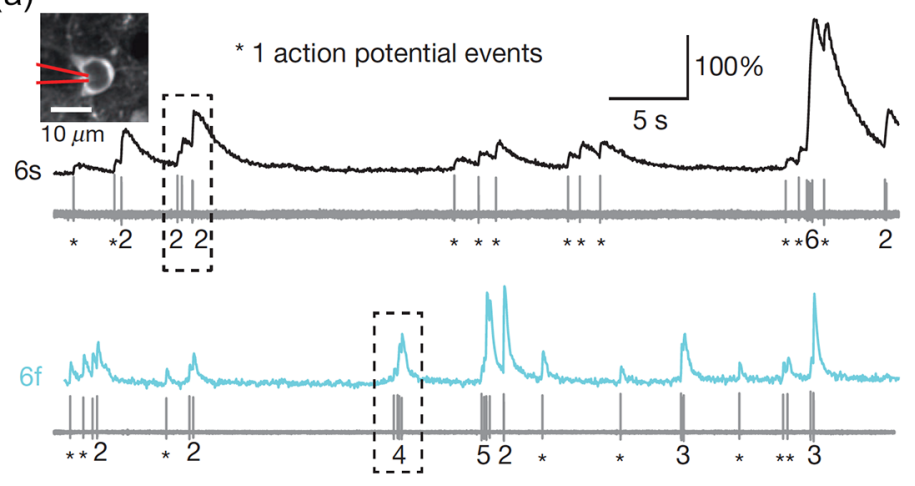

(b)

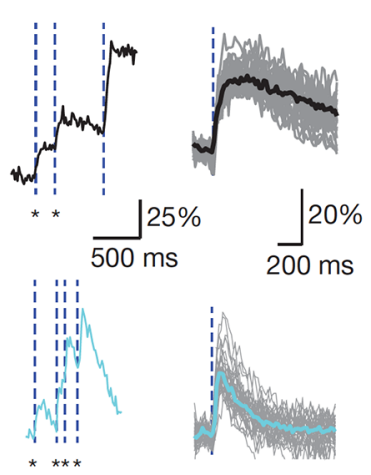

Fig. 1 Somatic calcium transients reflect spiking activity of cortical neurons. (a) Somatic calcium transients from a GCaMP6s- (top row) or GCaMP6f-expressing neuron (bottom row) in layer 2/3 of the mouse visual cortex, imaged using a two-photon microscope. Spiking activity was recorded from the imaged neurons using a juxtacellular electrode. The numbers denote the number of action potentials. An asterisk denotes a single spike. (b) Magnified view of the boxes in (a). (c) Median fluorescence transient in response to single action potential events with nearby spikes at least $1 \mathrm{~s}$ away. Figure adapted from Ref. 6. Reproduced with permission, courtesy of Springer Nature. 
In a few cases, such as granule cells in the Xenopus tadpole olfactory bulb ${ }^{38}$ and principal neurons in the locust antennal lobe ${ }^{39}$ the somatic calcium signals do not strictly follow the spiking activity. This suggests that additional sources of calcium, such as low-threshold calcium channels or internal stores, are contributors and cannot be neglected for the soma of those cell types. These counterexamples highlight the need to calibrate for the cell type and animal model of interest.

\section{Somatic Calcium Signals: for Neurons with High Firing Rates}

Some neurons, such as the GABAergic fast-spiking cells in the neocortex, have high firing rates. Although spike inference becomes more difficult, calcium imaging may still be an attractive approach because some interneuron subtypes are few in numbers and sparsely distributed and, therefore, difficult to record with other methods. Applications of interneuron imaging include determining the orientation selectivity of GABAergic neuron subtypes in the rodent visual cortex ${ }^{40}$ and dissecting functional responses of interneuron subtypes during delayed response tasks. ${ }^{41}$

Several years ago, we performed simultaneous imaging and electrophysiological recording to provide evidence for a direct relationship between somatic calcium signals and firing rates in cortical GABAergic neurons $^{32}$ (Fig. 2). Certain interneurons, such as parvalbumin-expressing (PV) fast-spiking cells, have a high in vivo firing rate-up to an instantaneous rate of $\sim 35 \mathrm{~Hz}$ in anesthetized mice in our study and a mean rate of $\sim 50 \mathrm{~Hz}$ in awake, behaving mice. ${ }^{42,43}$ For several reasons, as detailed at the end of this section, inferring the times of individual action potentials was imprecise in our original study. Nevertheless, most PV cells exhibited fluorescence signals that closely tracked the firing rate [Fig. 2(a)]. Therefore, even in a case when individual spikes could not be resolved, calcium imaging may be used to read out changes in firing rate.

Perhaps the most notable insight from those experiments was the variability. There was variability across cells belonging to the same neuron subtype. For example, the $10 \mathrm{PV}$ interneurons had substantial cell-to-cell variability in terms of fluorescence change per action potential [Fig. 2(b)]. This may be due to differences in the concentration of calcium indicators, whether through uneven loading of synthetic dyes or differential expression levels of GECIs. There was also variability across the neuron subtypes. In particular, the fluorescence change per action potential was greatest for excitatory neurons, and $\sim 50 \%$ lower for somatostatin-expressing (SST) and PV interneurons [Figs. 2(c) and 2(d)]. These differences are probably due to endogenous calcium buffers in GABAergic interneurons. ${ }^{44,45}$

Linearity in the spike-to-fluorescence relationship is important. Namely, one action potential should induce the same increase in fluorescence signal, regardless of the baseline calcium level. On average, with the synthetic dye OGB-1, the three cortical neuron subtypes exhibited fluorescence signals that scaled linearly with firing rates [Fig. 2(d)]. For individual neurons, the majority of the PV cells had a linear response curve up to $\sim 30 \mathrm{~Hz}$, although in a few selected cells, saturation was apparent at high firing rates [Fig. 2(b)]. These observations are consistent with an independent in vivo calibration for PV interneurons. ${ }^{46}$ A linear spike-to-fluorescence relationship depends on having a linear calcium-to-fluorescence relationship. Unlike OGB-1, GECIs that rely on cooperative calcium binding, such as GCaMP6, have nonlinear response curves. ${ }^{23}$ If the saturation or nonlinear response profile is known, the distortion may be corrected to some extent in post hoc analysis. ${ }^{47}$

(a)
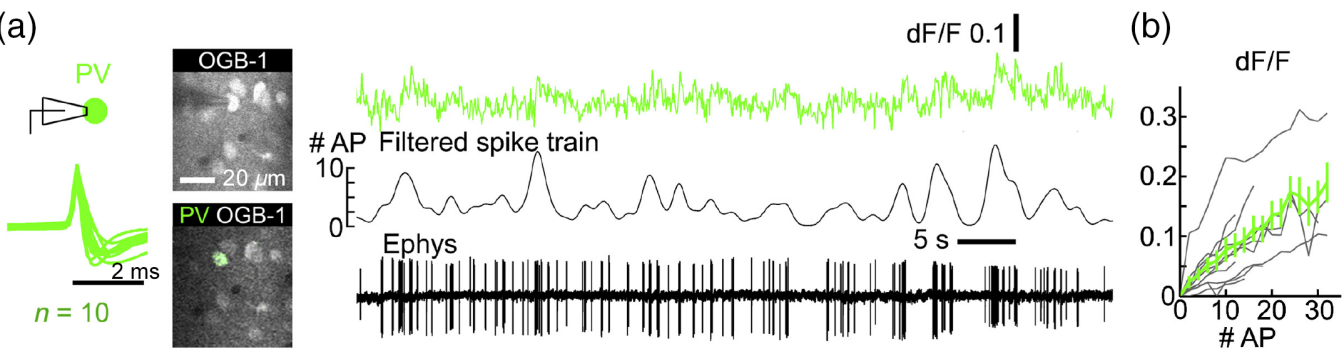

(c) Mean spike-triggered $d F / F$

(d) Mean $d F / F$ vs. spike rate
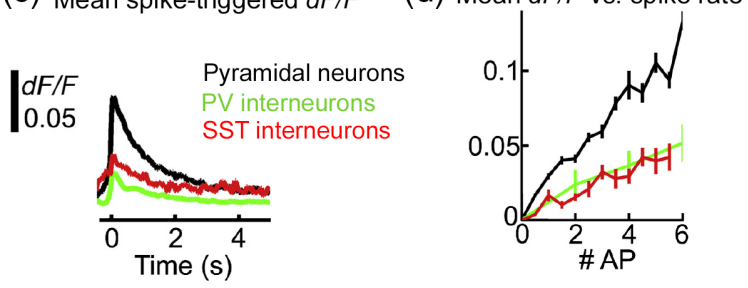

Fig. 2 Somatic calcium transients correlate with firing rates of cortical GABAergic neurons. (a) Somatic calcium transients from a parvalbumin-expressing (PV) interneuron in layer $2 / 3$ of the mouse visual cortex, imaged using a two-photon microscope. Cell type was identified based on tdTomato expression. Neurons were loaded with the synthetic calcium dye Oregon Green BAPTA-1 (OGB-1). Spiking activity was recorded under cell-attached condition. The left panel shows the mean normalized spike waveforms of 10 cells. The middle panel shows images including a recorded neuron. The right panel shows fluorescence and electrophysiological traces from an example cell. The filtered spike train was smoothed with a Gaussian filter (s.d. = $0.5 \mathrm{~s}$ ). (b) Fluorescence versus number of action potentials was determined using the filtered spike train. Gray lines, individual cells. Black line, mean \pm s.e.m. (c) Mean spiketriggered fluorescence for excitatory $(n=16)$, PV $(n=10)$, and SST neurons $(n=8)$. (d) Mean fluorescence versus number of action potentials for excitatory, PV, and SST neurons. The figure is adapted from Ref. 32. Reproduced with permission, courtesy of Elsevier. 
Inferring individual spikes under the high firing rate condition is a challenging problem. Important factors include imaging speed, indicator kinetics, and signal-to-noise ratio. The need for high imaging speed is obvious, as the sampling rate must be faster than the frequency content of the underlying continuous signal to obey the sampling theorem. Indicator kinetics, including rise and decay times, are also crucial. When kinetics is slow relative to spike rate, fluorescence responses to adjacent spikes overlap and cannot be discerned. Signal-to-noise ratio-a factor of the indicator and experimental condition-must be sufficient to detect the fluorescence change due to a single spike. Optimization of these parameters and their interactions is a complex topic, as detailed in a prior study with numerical simulations, ${ }^{47}$ and drives GECI engineering efforts. ${ }^{23,48}$

\section{Somatic Calcium Signals: for Measuring a Decrease in Firing Rate}

The somatic calcium transient due to an action potential has a near-instantaneous rise and then a relatively long decay. How much does the extended time course obscure the detection of a sudden decrease in firing rate? Figure 2(a) suggests that such decreases should be detectable. In that example, the PV interneuron has a high baseline activity in vivo, and fluorescence signals could track fluctuations above and below the median firing rate.

There are more explicit demonstrations that calcium signals can report a reduction in firing rate. In one experiment, a PV interneuron co-expresses an opsin for photoinhibition as well as a redshifted calcium sensor in vivo [Fig. 3(a)]. The effect of photoinhibition was confirmed by the suppressed spiking activity recorded by a juxtacellular electrode. For a single trial involving a 5-s long silencing period, there was an obvious dip in the calcium signal when spikes were suppressed [red-shaded area, Fig. 3(a)].

A more thorough experimental demonstration of how calcium signals relate to reduced firing rate has been done in vitro in acute brain slices (see extended data Fig. 2 in Ref. 50). In particular, that study showed that a decrease in spike rate leads to a negative calcium transient, but hyperpolarization of the membrane potential without any action potential change had no effect on the calcium signal. This is expected because hyperpolarization should have minimal influence on the permeability

(a) Soma-targeted GtACR2 + jRCaMP1a in layer $2 / 3$ $\mathrm{PV}^{+}$cells
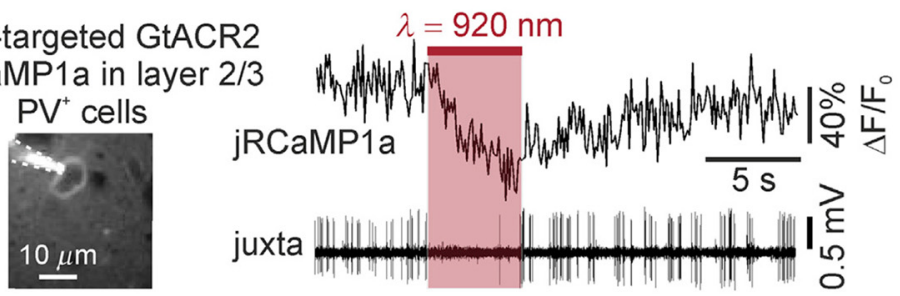

(b) Numerical simulation

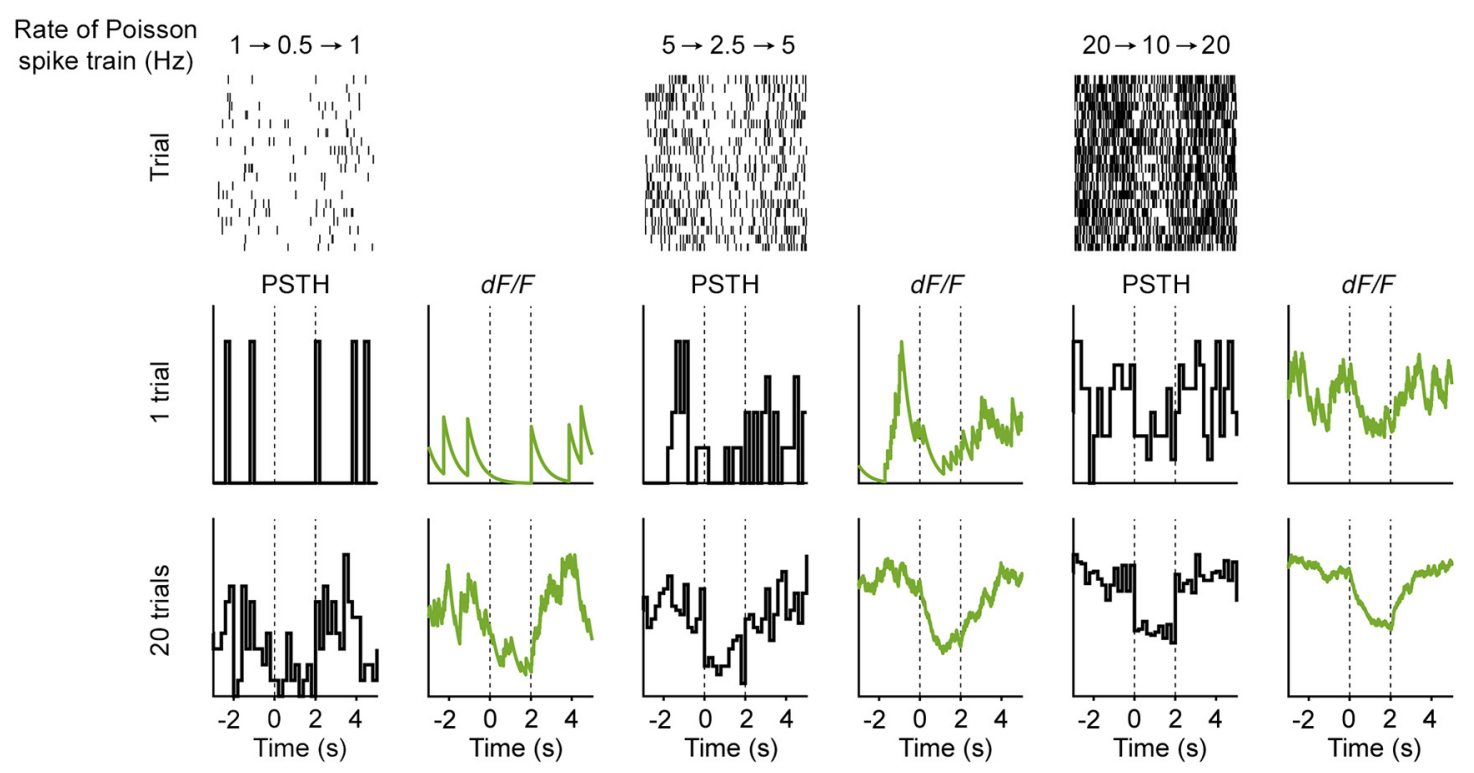

Fig. 3 Somatic calcium signals can report a decrease in firing rate. (a) A layer 2/3 PV interneuron expresses the redshifted calcium indicator jRCaMP1a and the soma-targeted opsin GtACR2. Traces show the fluorescence and spiking activity for the neuron in vivo. The red-shaded area denoted the time of the illumination to induce inhibition. (b) In these numerical simulations, for each trial, spike times were generated as a Poisson process, in which the rate parameter was halved at $t=0 \mathrm{~s}$ and then returned to baseline at $t=2 \mathrm{~s}$. The calcium response to a spike was approximated as a single-exponential function with a decay time constant of $0.55 \mathrm{~s}$. To determine the fluorescence signal, the spike-induced calcium transients were summed linearly. Peristimulus time histograms (PSTH) were generated by counting spikes in 200-ms bins. The range of the vertical axis for PSTH and fluorescence signals was arbitrary and rescaled for each plot. Panel (a) is adapted from Ref. 49. Panel (b) is unpublished data from the Kwan lab. 
of the calcium channels in the cell body. In other words, as a measure of inhibition, calcium cannot be used to detect inhibition in the form of hyperpolarization without spiking activity.

The same question can be answered with numerical simulations. For Fig. 3(b), we simulated how the somatic calcium signals would look if spike rate is halved for cells with varying amount of baseline firing. Notably, a reduction in calcium signal is obvious in single trials for neurons with high baseline activity, or by averaging across multiple trials for a wider range of firing rates. The more difficult cases are to discern a decrease in fluorescence if the neuron has low baseline firing, small reduction in spike rate, or only a few trials. However, this problem is not unique to calcium imaging, as peristimulus time histograms for spiking data is also noisy under these conditions [Fig. 3(b)]. And as is the case for spike inference, the sensitivity of detecting a reduction in firing rate would also depend on indicator kinetics and signal-to-noise ratio.

\section{Axonal Calcium Signals: as a Proxy for Presynaptic Activity}

Calcium signals from axonal boutons reflect depolarization in the presynaptic terminals and therefore can provide information about the afferent activity. When applied to long-range projections, axonal calcium imaging has been used to study the flow of information from the primary visual cortex to higher visual areas, ${ }^{51}$ determine the behavioral variables encoded by different afferent inputs to the motor cortex,${ }^{52}$ and map the spatial organization of long-range feedback projections. ${ }^{53}$

Briefly, membrane depolarization at an axonal terminal leads to calcium influx. For example, in layer $2 / 3$ pyramidal neurons, calcium entry near the presynaptic active zone is mediated by voltage-dependent calcium channels including P/Q- and Nsubtypes. ${ }^{54,55}$ Calcium signals are larger at the synaptic terminals than the flanking axonal segments, presumably due to a nonuniform distribution of calcium channels. The presynaptic calcium influx for a single action potential was estimated to have a fast rise time of $\sim 1 \mathrm{~ms}$ and a decay time constant of $\sim 60 \mathrm{~ms}$. An axonal calcium transient could be reliably elicited every time by an action potential, with an estimated mean increase of $500 \mathrm{nM}$ at the terminal. Interestingly, when responses to a single action potential were measured across multiple axonal boutons from the same neuron, there was a more than 10-fold variation in the intensity of calcium transients. ${ }^{56}$ This variability across boutons did not depend on distance from the soma but may instead depend on the postsynaptic cell type.$^{57}$ Calcium dynamics in the presynaptic terminals are also influenced by neuromodulators such as adenosine ${ }^{55}$ and dopamine. ${ }^{58}$

We have conducted experiments to assess the correlation between axonal calcium levels and afferent activation in an awake mouse $^{59}$ (Fig. 4). For these experiments, we targeted neurons in the retrosplenial cortex (RSC), which send dense axonal projections to the cingulate and secondary motor cortical regions (Cg1/M2) ${ }^{60,61}$ An adeno-associated virus was used to express GCaMP6s in RSC neurons. We then imaged their axons in $\mathrm{Cg} 1 / \mathrm{M} 2$ while simultaneously stimulated RSC using bipolar electrodes [Fig. 4(a)]. In response to raising levels of electrical stimulation, there was a graded increase in the evoked fluorescence transients from axonal boutons [Figs. 4(b) and 4(c)]. The response profile to afferent activation is consistent with a previous in vitro calibration done in brain slices (see Supplementary Fig. 2 in Ref. 52). Altogether, these results provide evidence that the calcium signals recorded from axonal compartments in vivo is related to depolarization of the afferents.

Because prior study has suggested substantial variability in calcium responses across boutons from the same cell, attempts to use data from a single axonal bouton to infer the firing rate of the presynaptic cell would be prone to large errors. Furthermore, the dependences of the axonal calcium influx on the postsynaptic cell type and neuromodulators mean that an aggregate analysis including many boutons would bias toward subpopulations of terminals with larger calcium amplitudes. These are issues that will affect the interpretations until we gain a deeper understanding of the factors that influence calcium elevations in the axon.

\section{Axonal Calcium Signals: as a Correlate of Neuromodulator Release}

Axonal calcium signals may relate to neuromodulator release, particularly if calcium indicators are expressed in principal cells of a neuromodulatory system. For example, calcium signals can be imaged from cholinergic axons to investigate the role of acetylcholine during active behavior, ${ }^{62,63}$ from cholinergic and noradrenergic axons to study pupil-related arousal, ${ }^{64}$ and (a)

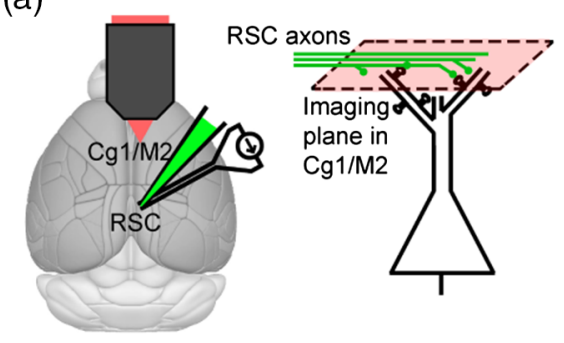

(b)

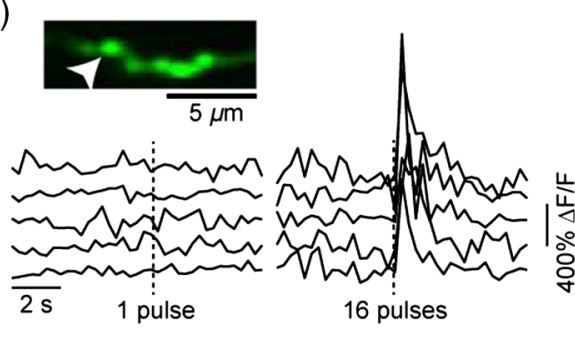

(c)

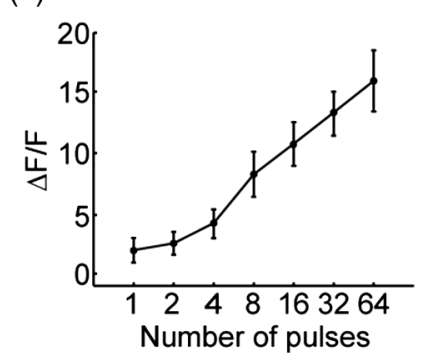

Fig. 4 Axonal calcium signals as a function of presynaptic neuronal activation. (a) Schematic of the experimental setup. Viruses were injected to express GCaMP6s in neurons in the RSC. Bipolar stimulating electrodes were implanted also in the RSC. Imaging was done in the Cg1/M2 to visualize the longrange axons from RSC neurons. (b) Top, in vivo two-photon image of a GCaMP6s-expressing axonal segment. Arrowhead, the example bouton analyzed. Bottom, $\Delta F / F$ traces from the example bouton in response to either 1 or 16 current injection pulses $( \pm 150 \mu \mathrm{A}, 10 \mathrm{~ms}$ per pulse) per trial. Five trials were shown for each stimulation strength. (c) The trial-averaged $\Delta F / F$ response within $1 \mathrm{~s}$ of the stimulation as a function of the stimulation strength, for the example bouton in (b). Line, mean $\pm \mathrm{SEM}$. These are unpublished data from the Kwan lab, related to a recent study. ${ }^{59}$ 
from dopaminergic axons to relate to locomotion and rewardbased behavior. ${ }^{65}$ In these examples, neuromodulator release might not have been explicitly inferred, but nevertheless the calcium signals are often interpreted by comparing with previous studies that measure extracellular concentrations of the neuromodulators using methods such as microdialysis or fast-scan cyclic voltammetry.

At least one study has empirically tested the relation between calcium signals and evoked neuromodulator release. ${ }^{66}$ Stimulation of the medial forebrain bundle leads to the release of dopamine in the striatum. This is an ideal testbed because the evoked dopamine transient is large. In this study, a carbon-fiber microelectrode was inserted into the striatum for cyclic voltammetry measurements of extracellular dopamine concentration [Figs. 5(a) and 5(b)]. Simultaneously, an optical fiber was inserted nearby to record the summed fluorescence signal of many dopaminergic axonal fibers. Relative to the evoked elevation of dopamine, the fluorescence transient had a shorter latency to peak, and a narrower width at half maximum [Figs. 5(c)-5(e)]. Importantly, calcium signals had a decent dynamic range. That is, fluorescence was sensitive to a range of evoked dopamine concentrations, and only began to saturate at the highest stimulation strengths for the largest evoked dopamine transients [Fig. 5(f)].

There are a number of caveats relating axonal calcium signals to neuromodulator release. Figure 5 shows that the calcium signals can track rapid phasic changes in dopamine concentration; however, the tonic level may not be detectable, as the calcium indicator can report relative changes but not the absolute amount of axonal activity. Furthermore, although an action potential would reliably depolarize the presynaptic terminal and cause calcium influx, the next steps in the chain of events involve the probabilistic release of a synaptic vesicle, and then the discharge and diffusion of several thousand neuromodulator molecules from a vesicle into extracellular space. As a result, the inference of neuromodulator levels from axonal calcium signals is complicated by additional, stochastic steps. Thus, although in vivo evidence suggests that the inference is reasonable when averaging across many axons and numerous trials, the correlation is likely much weaker at the single-axon level.

\section{Dendritic Calcium Signals: as a Readout of Backpropagating Action Potentials}

In dendrites, membrane depolarization can reflect a variety of passive and active electrical processes. ${ }^{17,18}$ One important source of dendritic calcium elevations is the backpropagating action potential (bAP). A bAP occurs because an action potential initiated close to the soma can travel bidirectionally, such that it would not only continue along the axon but also propagate backward into the dendritic tree. As a retrograde signal of neuronal output to the dendritic tree, the bAP may play important roles in synaptic plasticity and associative learning. ${ }^{67}$ (a)

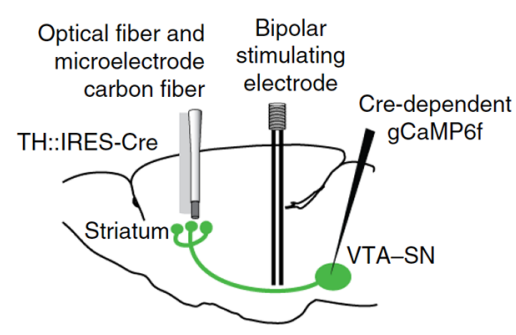

(d)

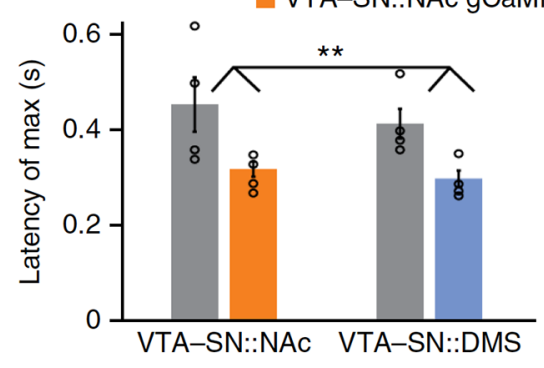

(b)

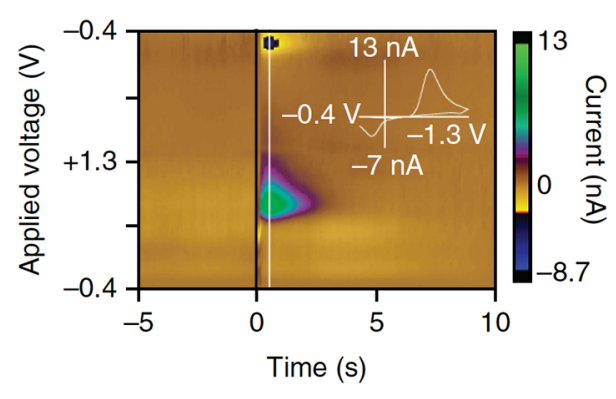

(e)

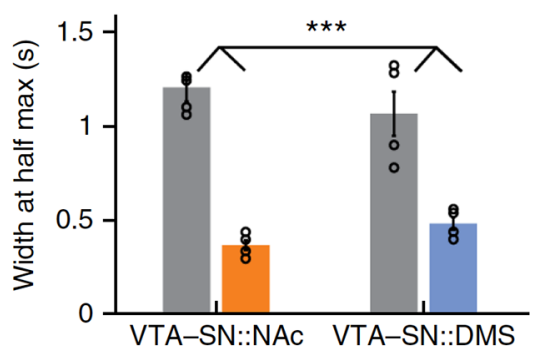

(c)
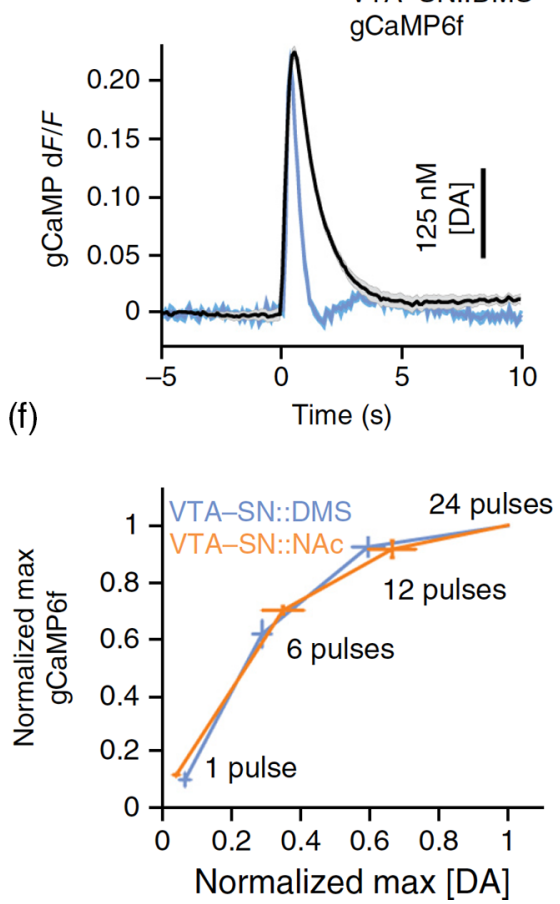

Fig. 5 Axonal calcium signals correlate with evoked dopamine release. (a) The medial forebrain bundle was stimulated to evoke dopamine release in a mouse. In the nucleus accumbens (NAc) or dorsomedial striatum (DMS), an optical fiber and a carbon-fiber microelectrode were inserted for photometric measurements of calcium and cyclic voltammetry of extracellular dopamine, respectively. Calcium signals arise from axons of GCaMP6f-expressing dopaminergic neurons in the ventral tegmental area and substantia nigra (VTA-SN). (b) Example recording of evoked dopamine efflux using cyclic voltammetry. (c) Comparison of the trial-averaged axonal calcium signal with the recorded dopamine transient. (d), (e) The latency and width of the calcium and dopamine signals, plotted separately for VTA-SNto-NAc and VTA-SN-to-DMS axons. (f) The peak calcium signals as a function of the peak evoked dopamine levels for various stimulation strengths. Figure is adapted from Ref. 66. Reproduced with permission, courtesy of Springer Nature. 
Propagation of the bAP relies on active conductances including voltage-activated sodium channels. ${ }^{68}$ In the absence of synaptic inputs, calcium can enter the dendritic shafts and spines through voltage-gated calcium channels. ${ }^{54}$ The channel subtypes involved vary depending on the cell type. ${ }^{17}$ The bAP is present in a variety of cell types, such as cortical pyramidal neurons, ${ }^{68}$ cortical GABAergic bitufted interneurons, ${ }^{69}$ and striatal medium spiny neurons, ${ }^{70}$ but is much attenuated in other cell types, such as cerebellar Purkinje cells. ${ }^{71}$

Experiments that combine imaging with electrophysiology have demonstrated dendritic calcium transients that are coincident with action potentials recorded in the soma. ${ }^{72,73}$ The more ideal and causal test would be to elicit action potentials by injecting current into the soma. ${ }^{74-76}$ In one study, ${ }^{77}$ in an anesthetized mouse, a layer $2 / 3$ neuron in the visual cortex was targeted for whole-cell recording while a two-photon microscope monitors fluorescent signals in the proximal dendritic segments [Fig. 6(a)]. Action potentials were evoked by current injection into the cell body. Although the bAP is around a millisecond in duration, the fluorescent transient is prolonged due to the life cycle of calcium and indicator kinetics. The fluorescent signals were linearly associated with the number of action potentials [Fig. 6(b)]. The bAP-associated calcium signal spread along the proximal dendrite with noticeable attenuation at the farthest imaged locations [Fig. 6(c)].

The amount of bAP-associated calcium influx is dependent on a number of factors. One important factor is the distance from the initiation site of the action potential. In anesthetized animals, for layer $2 / 3$ pyramidal neurons, the bAP attenuates and broadens as a function of distance from the soma, such that the calcium elevation becomes negligible beyond 200 to $250 \mu \mathrm{m}$. ${ }^{74,75}$ For layer 5 pyramidal neurons, beyond the main bifurcation point of the apical tuft, calcium influx is absent or unreliable for single bAP. ${ }^{76}$ Distance is the determining factor, because unlike the apical tuft, there is considerable correlation between somatic firing and calcium transients in the more proximal apical trunk and in the basal dendrites of these neurons. ${ }^{73}$ Other factors include differences in the morphology of the dendritic tree $^{78}$ or the distribution of dendritic voltage-gated channels.

\section{Dendritic Calcium Signals: as a Readout of Dendritic Regenerative Events}

A regenerative event may be initiated at the dendrites in response to the temporally synchronous and spatially clustered activation of many synaptic inputs. The resulting broad dendritic spike is accompanied by large-amplitude calcium influx. Calcium entry may be mediated through $N$-methyl-D-aspartate (NMDA)-type glutamate receptors and voltage-gated calcium channels. Their relative contribution is thought to depend on the dendritic location. ${ }^{79,80}$ For example, in layer 5 pyramidal neurons in vitro, calcium elevations from dendritic spikes could be confined to the activated branch in the most distal, fine tuft dendrites for NMDA spikes ${ }^{79}$ or spread to $\sim 50$ to $150 \mu \mathrm{m}$ from the initiation site in the apical tuft near the main bifurcation point for calcium spikes. ${ }^{81-83}$ Unlike sodium action potentials, the depolarization from calcium spikes can last hundreds of milliseconds.

Simultaneous calcium imaging and dendritic patch-clamp recording of layer 5 pyramidal neurons have provided evidence for compartmentalized calcium elevations that are regulated locally in dendrites in vivo. An early in vivo study showed that dendritic calcium events and somatic action potentials are not always coupled-namely, sometimes there is somatic burst spiking but no detectable change in dendritic calcium, whereas other times there is only one spike but a large dendritic calcium transient near the main bifurcation point of the apical dendrite. ${ }^{76}$ In a more recent study, ${ }^{84}$ it was shown that moderate dendritic voltage events in the apical trunk were not associated with any detectable calcium signals in the distal tuft dendrites in an anesthetized mouse [boxes 1 and 2 in Figs. 7(a)-7(d)]. However, (a)

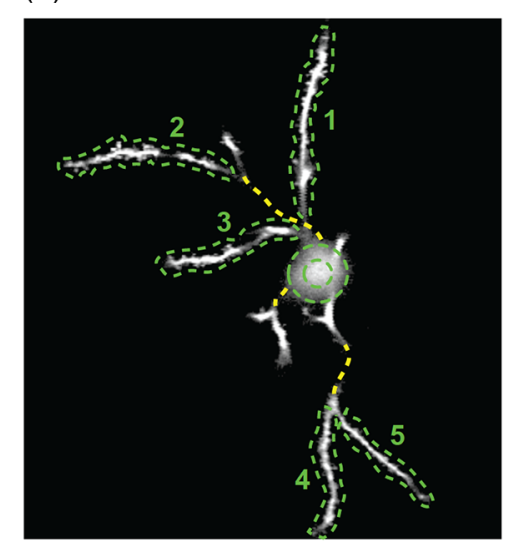

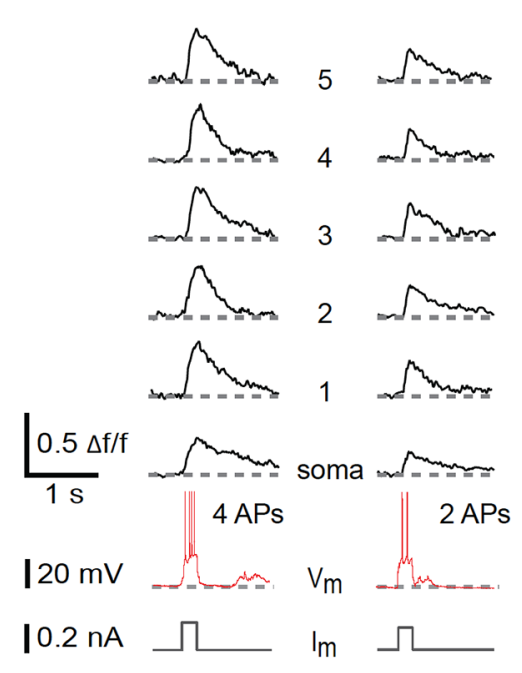

(b)

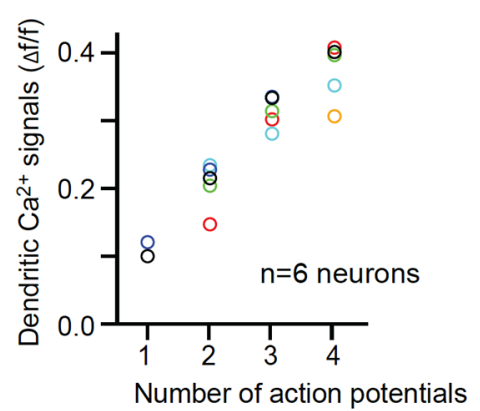

(c)

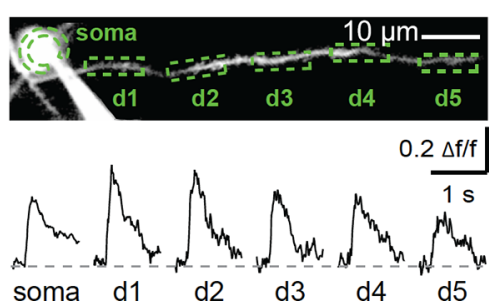

Fig. 6 Dendritic calcium signals from bAPs. (a) The left panel shows an in vivo two-photon image of a layer 2/3 neuron in the visual cortex loaded with OGB-1. The cell was also targeted for whole-cell recording. Green lines denote the dendritic regions analyzed. The right panel shows fluorescence traces for each of the dendritic region in response to somatic current injection producing either four or two action potentials. (b) The mean amplitude of dendritic calcium signals versus the number of evoked action potentials. Each circle denotes a cell. (c) Fluorescence responses in a dendrite as a function of distance from soma. Figure is adapted from Ref. 77. Reproduced with permission, courtesy of Springer Nature. 
(a)

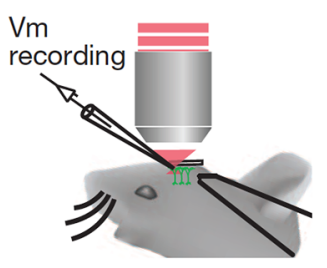

(b)

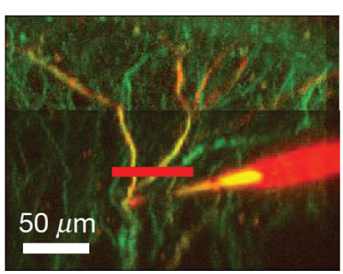

(c)

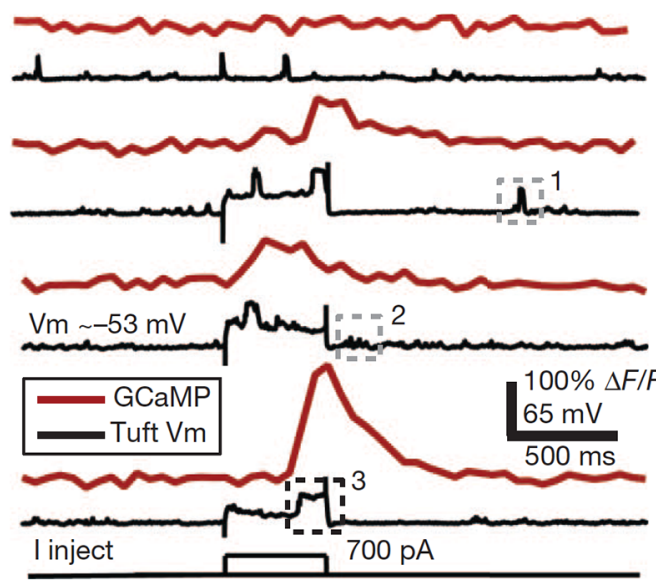

(d)

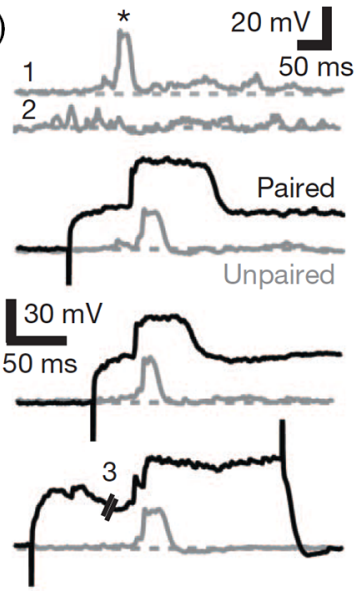

Fig. 7 Dendritic calcium signals from dendritic regenerative events. (a) Schematic of the experiment involving in vivo two-photon imaging and dendritic patch-clamp recording in an anesthetized mouse. (b) A layer 5 neuron in the barrel cortex expresses GCaMP3 (green). The patch pipette was filled with Alexa Fluor 594 (red). (c) The membrane potential at the apical dendrite (black) was plotted along with calcium signals (red) imaged in line-scan mode as indicated by the red line in (b). The top voltage trace is from a trial without current injection. The lower three voltage traces are from trials with a 500-ms, 700-pA current step. (d) Magnified view of the dashed boxes in (c). The gray traces are spontaneous dendritic voltage events. The black traces show plateau potentials during the paired events in which current was injected. Figure is adapted from Ref. 84. Reproduced with permission, courtesy of Springer Nature.

when a depolarizing current step was injected at the apical trunk, the coincident arrival of a spontaneous voltage event would induce a longer-lasting plateau depolarization, which was accompanied by a large-amplitude calcium transient in tuft dendrites [box 3 in Figs. 7(c) and 7(d)]. Critically, the current step alone was not sufficient to induce detectable calcium signals in the distal tuft. The interpretation for these data is that the dendritic calcium transients were due to occurrences of regenerative plateau potentials in the apical compartment, on the basis of the stimulation location. Intriguingly, this study presented relatively widespread dendritic calcium signals across multiple distal tuft branches, which differs from a couple of other accounts of transients that are typically more spatially restricted ${ }^{73,85}$ or branchspecific. ${ }^{86}$ One challenge is that without the knowledge of the calcium signals in the apical trunk, some of the putative branchspecific signals may in fact be more global plateau potentials that have not invaded all branches, and thus masquerade as compartmentalized calcium transients. A recent study estimated only about $15 \%$ of the calcium signals in distal tufts are independent from those in the apical trunk in layer 5 pyramidal neurons during awake behavior. ${ }^{87}$ Pharmacological manipulations could provide additional clues as to the origin of these dendritic calcium signals in vivo ${ }^{85,88}$ Nevertheless, more work is needed to unequivocally attribute in vivo dendritic calcium transients to specific types of nonlinear regenerative events.

\section{Dendritic Calcium Signals: as a Readout of Synaptic Input}

Dendritic spines are protrusions on the dendritic shaft. Most dendritic spines are functional glutamatergic synapses that receive excitatory inputs. Upon depolarization from an excitatory input, calcium ions enter the spine compartment with minimal outflow to the dendritic shaft. ${ }^{89,90}$ The localized signal is unlike the situation for bAP in which calcium invades both spines and the connecting shaft. ${ }^{54}$ For pyramidal neurons, a large portion of the synaptically evoked calcium influx is probably driven by NMDA receptors, ${ }^{54,91}$ with some contributions by voltage-gated calcium channels. ${ }^{92,93}$ Because the localized calcium transient is a correlate of synaptic activation, spine imaging in vivo is a powerful method to characterize the organization of synaptic inputs, for example, to understand how inputs are integrated to give rise to feature selectivity in visual cortical neurons. ${ }^{77,94}$

Because subthreshold synaptic activation-one that occurs in the absence of somatic action potentials or other regenerative events-induces only localized influx, any calcium transient detected in the shaft and spine at the same time is likely due to other mechanisms such as bAP or dendritic spikes. Therefore, a common practice is to isolate the local component by removing those fluorescent transients that co-occur in the shaft and spine in post hoc analysis using a subtraction procedure. ${ }^{6,95,96}$ As an additional benefit, noises that cofluctuate in the shaft and spine, for example, from motion artifact, would be reduced by the subtraction procedure. How well does the procedure work? To answer this question, we have imaged spontaneous calcium transients in apposing bouton-spine pairs in awake mice. More specifically, we expressed GCaMP6s in RSC excitatory neurons and the redshifted calcium sensor jRGEOC1a in Cg1/M2 neurons [unpublished data; Fig. 8(a)]. In Cg1/M2, putative connected bouton-spine pairs could be identified based on proximity [Fig. 8(b)]. We followed a typical subtraction procedure, by using linear regression to estimate the shaft-spine signal ratio, which allows us to scale the shaft signal and subtract its contribution from the spine signal [Fig. 8(c)]. As expected for functional synapses, the subtraction-isolated, local fluorescent transients from the dendritic spine were highly correlated with signals from the axonal bouton [Fig. 8(d)]. To quantify the relationship, we converted the fluorescence transients into calcium events using a peeling algorithm, ${ }^{47}$ and then calculated the conditional probability of observing a presynaptic event given a postsynaptic event. In an example bouton-spine pair, we estimated $\sim 70 \%$ of the postsynaptic events imaged in the dendritic spine were coincident with synaptic input [Fig. 8(e)]. By contrast, the estimate fell to $\sim 30 \%$ when the subtraction procedure 
(a)

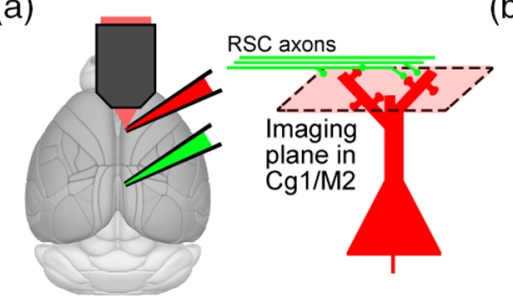

(b)

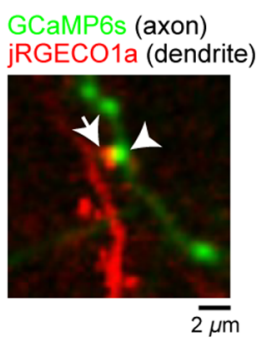

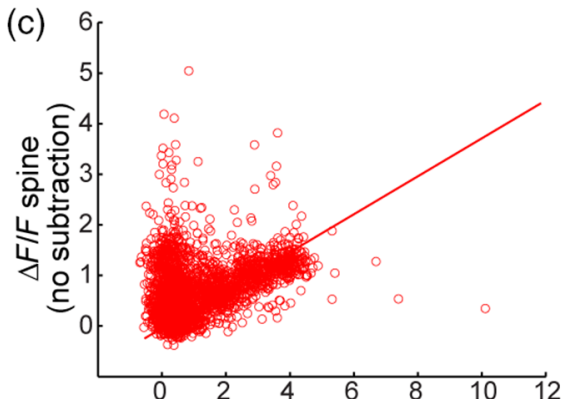

$\Delta F / F$ dendritic shaft (d)

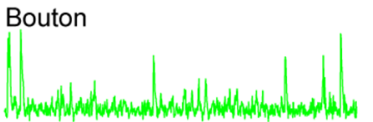

Apposing spine (with subtraction)

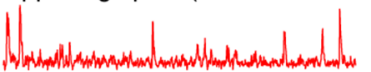

Apposing spine (no subtraction)

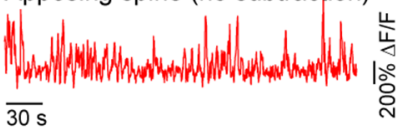

$(e)$

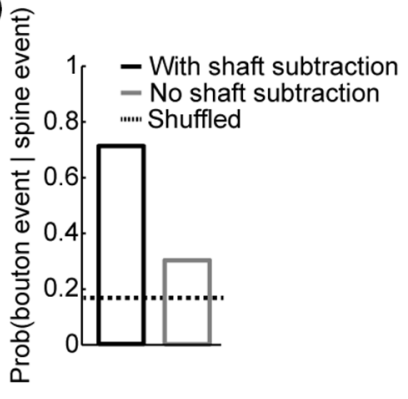

Fig. 8 Dendritic calcium signals from synaptic inputs. (a) The experimental setup involving expression of GCaMP6s in RSC and jRGECO1a in Cg1/M2. (b) An in vivo two-photon image of GCaMP6s-expressing axonal boutons and jRGECO1a-expressing dendrites in Cg1/M2. (c) A scatter plot of the fluorescence transients $(\Delta F / F)$ measured from spine indicated by arrow in (b) against the $\Delta F / F$ measured from the adjacent dendritic shaft. Each open circle represents an image frame. Line, a least-squares regression line forced through the origin. (d) Fluorescence traces for the bouton and apposing spine (either with subtraction or no subtraction of the shaft contribution) in (b). (e) The probability of detecting a presynaptic calcium event in the bouton within $\pm 0.276 \mathrm{~s}$ (i.e., \pm the duration of one image frame) given a postsynaptic calcium event in the apposing spine, either with subtraction or no subtraction of the shaft contribution. Calcium events were determined from fluorescence traces using a peeling algorithm. Shuffled level was calculated by randomly shuffling the calcium event times of the bouton, and averaged across 100 replicates. These are unpublished data from the Kwan lab.

was not used, suggesting greater nonsynaptic contributions to the spine calcium signal. Isolation of the local component may be improved if the regression considers the distinct calcium diffusion and decay characteristics in the shaft and spine compartments. $^{87}$

One caveat of the aforementioned subtraction method is that, by definition, the procedure removes synaptic inputs that correlate with the occurrences of dendritic spikes and bAPs. Arguably those may include the strongest and clustered patterns of inputs that affect spiking activity and synaptic plasticity. To mitigate this issue, synaptic calcium signals may be imaged in the absence of spiking by hyperpolarizing the cell body or usingpharmacology. ${ }^{77,97}$ This would prevent bAP, although dendritic regenerative events may still occur in the distal compartment due to the space-clamp problem. Another caveat is that calcium enters dendritic spines predominantly through NMDAR, so we would report only those synaptic activations that are sufficiently strong to relieve the magnesium block. Because of these challenges in detecting the strongest and weakest activations, synaptic calcium signals are expected to report a fraction of the synaptic inputs arriving at a dendritic spine and should be interpreted accordingly.

\section{Putting It All Together}

From the viewpoint of an experimenter, current imaging techniques provide a limited picture of the entire neuronal structure. We typically visualize either the somatic, axonal, or dendritic compartment, and therefore it has been convenient to categorize calcium signals based on the imaging location. However, this classification ignores the dynamic nature of the physiology. Instead, if we consider from the vantage point of a neuron, a sodium action potential initiated near the soma would induce calcium influx in the cell body, and then would propagate and elevate calcium also in the axon and proximal dendrite. Or take the case of synaptic inputs, which initially elevate calcium in spines, but together a strong barrage of inputs could lead to a regenerative event that is accompanied by more widespread dendritic calcium signals. ${ }^{98,99}$ In a more complex situation, for example, in layer 5 pyramidal neurons, subthreshold synaptic inputs may pair with a bAP to drive plateau potentials and burst firing at the soma ${ }^{100}$ - a sequence of events that is expected to induce calcium influx in almost all compartments of a neuron.

Presumably the more complex sequences of physiological events are more prevalent during active behavior. This is because the strength of regenerative events such as the bAP is boosted by synaptic inputs, which are more frequent and powerful in vivo and in awake conditions. ${ }^{101,102}$ GABAergic neurons have complex firing patterns in vivo, and the strengthening or relief of inhibition could strongly influence calcium spikes ${ }^{103}$ and control calcium influx into dendritic spines. ${ }^{104}$ Indeed, dendritic calcium signals were found to be substantially enhanced in the same animal when awake rather than anesthetized. ${ }^{105}$ Putting it all together, instead of considering calcium elevations from isolable events in various compartments, one can move toward using the overall spatiotemporal pattern of calcium signals across the entire cell to infer the underlying spatiotemporal dynamics of electrical depolarization in the neuron. 


\section{Looking Ahead}

We need methods that can uncover calcium signals over a broader spatial scale. Some techniques, such as fiber photometry, provide no optical sectioning. Because calcium signals could come from a mix of somatic, axonal, and dendritic sources, the neural correlates should be interpreted with caution. One improvement is to use genetic strategies to restrict expression to the soma ${ }^{106}$ or axons, ${ }^{107}$ such that calcium indicators reside in only a specific cellular compartment; however, experiments would still suffer from the same problems as discussed in the last section.

To increase the spatial extent of imaging, emerging technologies enable data acquisition across multiple axial planes or from a volume. In this review article, most of the examples were acquired with two-photon microscopes. Using a high numericalaperture objective, the lateral resolution of this method is $\sim 0.5 \mu \mathrm{m}$ and axial resolution is $\sim 2 \mu \mathrm{m},{ }^{108}$ sufficient to visualize small compartments such as single dendritic spines. To maintain this spatial resolution but now over greater spatial extent, twophoton microscopes have incorporated design elements such as electrically tunable lens, ${ }^{109}$ Bessel focus, ${ }^{110}$ and remote focusing. ${ }^{87}$ Studies using these new microscopes have demonstrated rapid imaging of multiple neuronal compartments, such as numerous dendritic tuft branch locations along with the apical dendritic trunk or the cell body.

As the ability to report calcium transients and infer electrical events depends on the properties of the fluorescent indicator, the choice of the indicator should be matched to the needs of the experiment. For the latest generation of GECIs, this is exactly the rationale behind the multiple variants of the indicators. ${ }^{48,111}$ For example, the most sensitive variant is useful for the detection of single action potentials in the soma. By contrast, a variant with less sensitivity but brighter baseline fluorescence is desirable for dendritic imaging, because the higher basal signal is helpful for confirming the presence of a spine.

The indicator development, together with new imaging technologies, promises to deliver the full richness of calcium dynamics in neurons. This will help with addressing the current limitations of the calcium imaging technique and enable more accurate interpretations of the results.

\section{Disclosures}

The authors have declared that there is no conflict of interest.

\section{Acknowledgments}

All experimental procedures were approved by the Institutional Animal Care and Use Committee at Yale University. The section on "somatic calcium signals - for measuring a decrease in firing rate" was motivated by a discussion on Twitter initiated by Caitlin Vander Weele. This work was supported by the National Institute of Mental Health grants R01MH112750 (A.C.K.), R21MH110712 (A.C.K.), R21MH118596 (A.C.K.), National Institute on Aging grant P50AG047270 (A.C.K.), NARSAD Young Investigator Grant (A.C.K.), Simons Foundation SFARI Pilot Award (A.C.K.), Alzheimer's Association Research Fellowship AARF-17-504924 (F.A.), and James Hudson Brown-Alexander Brown Coxe Postdoctoral Fellowship (F.A.).

\section{References}

1. N. Takahashi et al., "Active cortical dendrites modulate perception," Science 354(6319), 1587-1590 (2016).
2. H. Makino et al., "Transformation of cortex-wide emergent properties during motor learning," Neuron 94(4), 880-890.e8 (2017).

3. M. J. Siniscalchi et al., "Fast and slow transitions in frontal ensemble activity during flexible sensorimotor behavior,' Nat. Neurosci. 19(9), 1234-1242 (2016).

4. J. B. Ackman, T. J. Burbridge, and M. C. Crair, "Retinal waves coordinate patterned activity throughout the developing visual system," Nature 490(7419), 219-225 (2012).

5. S. E. J. de Vries et al., "A large-scale, standardized physiological survey reveals higher order coding throughout the mouse visual cortex," bioRxiv 359513 (2019).

6. T. W. Chen et al., "Ultrasensitive fluorescent proteins for imaging neuronal activity," Nature 499(7458), 295-300 (2013).

7. H. Dana et al., "Sensitive red protein calcium indicators for imaging neural activity," Elife 5, e12727 (2016).

8. T. L. Daigle et al., "A suite of transgenic driver and reporter mouse lines with enhanced brain-cell-type targeting and functionality," Cell 174(2), 465-480.e22 (2018).

9. W. Denk, J. H. Strickler, and W. W. Webb, "Two-photon laser scanning fluorescence microscopy," Science 248(4951), 73-76 (1990).

10. N. G. Horton et al., "In vivo three-photon microscopy of subcortical structures within an intact mouse brain," Nat. Photonics 7(3), 205-209 (2013).

11. J. N. Stirman et al., "Wide field-of-view, multi-region, two-photon imaging of neuronal activity in the mammalian brain," Nat. Biotechnol. 34(8), 857-862 (2016).

12. E. Neher and G. J. Augustine, "Calcium gradients and buffers in bovine chromaffin cells," J. Physiol. 450, 273-301 (1992).

13. F. Helmchen, K. Imoto, and B. Sakmann, " $\mathrm{Ca}^{2+}$ buffering and action potential-evoked $\mathrm{Ca}^{2+}$ signaling in dendrites of pyramidal neurons," Biophys. J. 70(2), 1069-1081 (1996).

14. C. Grienberger and A. Konnerth, "Imaging calcium in neurons," Neuron 73(5), 862-885 (2012).

15. B. F. Grewe and F. Helmchen, "Optical probing of neuronal ensemble activity," Curr. Opin. Neurobiol. 19(5), 520-529 (2009).

16. S. Peron, T. W. Chen, and K. Svoboda, "Comprehensive imaging of cortical networks," Curr. Opin. Neurobiol. 32, 115-123 (2015).

17. M. J. Higley and B. L. Sabatini, "Calcium signaling in dendrites and spines: practical and functional considerations," Neuron 59(6), 902913 (2008).

18. C. Grienberger, X. Chen, and A. Konnerth, "Dendritic function in vivo," Trends Neurosci. 38(1), 45-54 (2015).

19. G. J. Augustine, F. Santamaria, and K. Tanaka, "Local calcium signaling in neurons," Neuron 40(2), 331-346 (2003).

20. G. J. Augustine, M. P. Charlton, and S. J. Smith, "Calcium action in synaptic transmitter release," Апnи. Rev. Neurosci. 10, 633-693 (1987).

21. R. Y. Tsien, "Monitoring cell calcium" in Calcium as Cellular Regulator, E. Carafoli and C. Klee, Eds., Oxford University Press, Oxford (1999).

22. F. Helmchen and D. W. Tank, "A single-compartment model of calcium dynamics in nerve terminals and dendrites," Cold Spring Harb. Protoc. 2015(2), 155-167 (2015).

23. T. Rose et al., "Putting a finishing touch on GECIs," Front. Mol. Neurosci. 7, 88 (2014).

24. C. Stringer and M. Pachitariu, "Computational processing of neural recordings from calcium imaging data," Curr. Opin. Neurobiol. 55, 22-31 (2019).

25. M. J. Siniscalchi, H. Wang, and A. C. Kwan, "Enhanced population coding for rewarded choices in the medial frontal cortex of the mouse," Cereb. Cortex bhy292 (2019).

26. K. Ohki et al., "Functional imaging with cellular resolution reveals precise micro-architecture in visual cortex," Nature 433(7026), 597-603 (2005).

27. H. Makino and T. Komiyama, "Learning enhances the relative impact of top-down processing in the visual cortex," Nat. Neurosci. 18(8), 1116-1122 (2015).

28. H. Markram, P. J. Helm, and B. Sakmann, "Dendritic calcium transients evoked by single back-propagating action potentials in rat neocortical pyramidal neurons," J. Physiol. 485(Pt. 1), 1-20 (1995).

29. J. Schiller, F. Helmchen, and B. Sakmann, "Spatial profile of dendritic calcium transients evoked by action potentials in rat neocortical pyramidal neurones," J. Physiol. 487(Pt. 3), 583-600 (1995). 
30. E. Neher, "Details of $\mathrm{Ca}^{2+}$ dynamics matter," J. Physiol. 586(8), 2031-2031 (2008)

31. J. N. Kerr, D. Greenberg, and F. Helmchen, "Imaging input and output of neocortical networks in vivo," Proc. Natl. Acad. Sci. U. S. A. 102(39), 14063-14068 (2005).

32. A. C. Kwan and Y. Dan, "Dissection of cortical microcircuits by single-neuron stimulation in vivo," Curr. Biol. 22(16), 1459-1467 (2012).

33. L. Tian et al., "Imaging neural activity in worms, flies and mice with improved GCaMP calcium indicators," Nat. Methods 6(12), 875-881 (2009).

34. J. T. Vogelstein et al., "Spike inference from calcium imaging using sequential Monte Carlo methods," Biophys. J. 97(2), 636-655 (2009).

35. B. F. Grewe et al., "High-speed in vivo calcium imaging reveals neuronal network activity with near-millisecond precision," Nat. Methods 7(5), 399-405 (2010).

36. P. Fries, "Modulation of oscillatory neuronal synchronization by selective visual attention," Science 291(5508), 1560-1563 (2001).

37. A. C. Kwan et al., "Spatiotemporal dynamics of rhythmic spinal interneurons measured with two-photon calcium imaging and coherence analysis," J. Neurophysiol. 104(6), 3323-3333 (2010).

38. B. J. Lin, T. W. Chen, and D. Schild, "Cell type-specific relationships between spiking and $\left[\mathrm{Ca}^{2+}\right] \mathrm{i}$ in neurons of the Xenopus tadpole olfactory bulb," J. Physiol. 582(Pt. 1), 163-175 (2007).

39. L. Moreaux and G. Laurent, "Estimating firing rates from calcium signals in locust projection neurons in vivo," Front. Neural Circuits 1, 2 (2007).

40. A. M. Kerlin et al., "Broadly tuned response properties of diverse inhibitory neuron subtypes in mouse visual cortex," Neuron 67(5), 858-871 (2010).

41. T. Kamigaki and Y. Dan, "Delay activity of specific prefrontal interneuron subtypes modulates memory-guided behavior," Nat. Neurosci. 20(6), 854-863 (2017).

42. D. Kim et al., "Distinct roles of parvalbumin- and somatostatinexpressing interneurons in working memory," Neuron 92(4), 902915 (2016).

43. H. Kim et al., "Prefrontal parvalbumin neurons in control of attention," Cell 164(1-2), 208-218 (2016).

44. S. H. Lee et al., "Differences in $\mathrm{Ca}^{2+}$ buffering properties between excitatory and inhibitory hippocampal neurons from the rat," $J$. Physiol. 525(2), 405-418 (2000).

45. Y. Aponte, J. Bischofberger, and P. Jonas, "Efficient $\mathrm{Ca}^{2+}$ buffering in fast-spiking basket cells of rat hippocampus," J. Physiol. 586(8), 2061-2075 (2008).

46. S. B. Hofer et al., "Differential connectivity and response dynamics of excitatory and inhibitory neurons in visual cortex," Nat. Neurosci. 14(8), 1045-1052 (2011).

47. H. Lutcke et al., "Inference of neuronal network spike dynamics and topology from calcium imaging data," Front. Neural Circuits 7, 201 (2013).

48. M. Inoue et al., "Rational engineering of XCaMPs, a multicolor GECI suite for in vivo imaging of complex brain circuit dynamics," Cell 177, 1346-1360.e24 (2019).

49. A. Forli et al., "Two-photon bidirectional control and imaging of neuronal excitability with high spatial resolution in vivo," Cell Rep. 22(11), 3087-3098 (2018).

50. J. M. Otis et al., "Prefrontal cortex output circuits guide reward seeking through divergent cue encoding," Nature 543(7643), 103-107 (2017).

51. L. L. Glickfeld et al., "Cortico-cortical projections in mouse visual cortex are functionally target specific," Nat. Neurosci. 16(2), 219-226 (2013).

52. L. Petreanu et al., "Activity in motor-sensory projections reveals distributed coding in somatosensation," Nature 489(7415), 299-303 (2012).

53. T. Marques et al., "The functional organization of cortical feedback inputs to primary visual cortex," Nat. Neurosci. 21(5), 757-764 (2018).

54. H. J. Koester and B. Sakmann, "Calcium dynamics in single spines during coincident pre- and postsynaptic activity depend on relative timing of back-propagating action potentials and subthreshold excitatory postsynaptic potentials," Proc. Natl. Acad. Sci. U. S. A. 95(16), 9596-9601 (1998).
55. C. L. Cox et al., "Action potentials reliably invade axonal arbors of rat neocortical neurons," Proc. Natl. Acad. Sci. U. S. A. 97(17), 97249728 (2000).

56. H. J. Koester and B. Sakmann, "Calcium dynamics associated with action potentials in single nerve terminals of pyramidal cells in layer $2 / 3$ of the young rat neocortex," J. Physiol. 529(Pt. 3), 625-646 (2000).

57. H. J. Koester and D. Johnston, "Target cell-dependent normalization of transmitter release at neocortical synapses," Science 308(5723), 863-866 (2005).

58. K. J. Burke, Jr., C. M. Keeshen, and K. J. Bender, "Two forms of synaptic depression produced by differential neuromodulation of presynaptic calcium channels," Neuron 99(5), 969-984.e7 (2018).

59. F. Ali et al., "Ketamine disinhibits dendrites and enhances calcium signals in prefrontal dendritic spines," bioRxiv 659292 (2019).

60. N. Yamawaki, J. Radulovic, and G. M. Shepherd, "A corticocortical circuit directly links retrosplenial cortex to M2 in the mouse," J. Neurosci. 36(36), 9365-9374 (2016).

61. F. Barthas and A. C. Kwan, "Secondary motor cortex: where 'sensory' meets 'motor' in the rodent frontal cortex," Trends Neurosci. 40(3), 181-193 (2017).

62. E. Eggermann et al., "Cholinergic signals in mouse barrel cortex during active whisker sensing," Cell Rep. 9(5), 1654-1660 (2014).

63. A. Nelson and R. Mooney, "The basal forebrain and motor cortex provide convergent yet distinct movement-related inputs to the auditory cortex," Neuron 90(3), 635-648 (2016).

64. J. Reimer et al., "Pupil fluctuations track rapid changes in adrenergic and cholinergic activity in cortex," Nat. Commun. 7, 13289 (2016).

65. M. W. Howe and D. A. Dombeck, "Rapid signalling in distinct dopaminergic axons during locomotion and reward," Nature 535(7613), 505-510 (2016).

66. N. F. Parker et al., "Reward and choice encoding in terminals of midbrain dopamine neurons depends on striatal target," Nat. Neurosci. 19(6), 845-854 (2016).

67. G. Stuart et al., "Action potential initiation and backpropagation in neurons of the mammalian CNS," Trends Neurosci. 20(3), 125-131 (1997).

68. G. J. Stuart and B. Sakmann, "Active propagation of somatic action potentials into neocortical pyramidal cell dendrites," Nature 367(6458), 69-72 (1994).

69. K. M. Kaiser, Y. Zilberter, and B. Sakmann, "Back-propagating action potentials mediate calcium signalling in dendrites of bitufted interneurons in layer $2 / 3$ of rat somatosensory cortex," J. Physiol. 535(Pt. 1), 17-31 (2001).

70. A. G. Carter and B. L. Sabatini, "State-dependent calcium signaling in dendritic spines of striatal medium spiny neurons," Neuron 44(3), 483-493 (2004).

71. R. Llinas and M. Sugimori, "Electrophysiological properties of in vitro Purkinje cell dendrites in mammalian cerebellar slices," J. Physiol. 305, 197-213 (1980).

72. K. Svoboda et al., "In vivo dendritic calcium dynamics in neocortical pyramidal neurons," Nature 385(6612), 161-165 (1997).

73. D. N. Hill et al., "Multibranch activity in basal and tuft dendrites during firing of layer 5 cortical neurons in vivo," Proc. Natl. Acad. Sci. U. S. A. 110(33), 13618-13623 (2013).

74. J. Waters et al., "Supralinear $\mathrm{Ca}^{2+}$ influx into dendritic tufts of layer $2 / 3$ neocortical pyramidal neurons in vitro and in vivo," J. Neurosci. 23(24), 8558-8567 (2003).

75. K. Svoboda et al., "Spread of dendritic excitation in layer $2 / 3$ pyramidal neurons in rat barrel cortex in vivo," Nat. Neurosci. 2(1), 65-73 (1999).

76. F. Helmchen et al., "In vivo dendritic calcium dynamics in deep-layer cortical pyramidal neurons," Nat. Neurosci. 2(11), 989-996 (1999).

77. H. Jia et al., "Dendritic organization of sensory input to cortical neurons in vivo," Nature 464(7293), 1307-1312 (2010).

78. P. Vetter, A. Roth, and M. Hausser, "Propagation of action potentials in dendrites depends on dendritic morphology," J. Neurophysiol. 85(2), 926-937 (2001).

79. M. E. Larkum et al., "Synaptic integration in tuft dendrites of layer 5 pyramidal neurons: a new unifying principle," Science 325(5941), 756-760 (2009).

80. J. Schiller et al., "NMDA spikes in basal dendrites of cortical pyramidal neurons," Nature 404(6775), 285-289 (2000). 
81. R. Yuste et al., " $\mathrm{Ca}^{2+}$ accumulations in dendrites of neocortical pyramidal neurons: an apical band and evidence for two functional compartments," Neuron 13(1), 23-43 (1994).

82. H. Markram and B. Sakmann, "Calcium transients in dendrites of neocortical neurons evoked by single subthreshold excitatory postsynaptic potentials via low-voltage-activated calcium channels," Proc. Natl. Acad. Sci. U. S. A. 91(11), 5207-5211 (1994).

83. J. Schiller et al., "Calcium action potentials restricted to distal apical dendrites of rat neocortical pyramidal neurons," J. Physiol. 505(Pt. 3), 605-616 (1997).

84. N. L. Xu et al., "Nonlinear dendritic integration of sensory and motor input during an active sensing task," Nature 492(7428), 247-251 (2012).

85. L. M. Palmer et al., "NMDA spikes enhance action potential generation during sensory input," Nat. Neurosci. 17(3), 383-390 (2014).

86. J. Cichon and W. B. Gan, "Branch-specific dendritic $\mathrm{Ca}^{2+}$ spikes cause persistent synaptic plasticity," Nature 520(7546), 180-185 (2015).

87. A. Kerlin et al., "Functional clustering of dendritic activity during decision-making," biorxiv 440396 (2018).

88. M. Murayama et al., "Dendritic encoding of sensory stimuli controlled by deep cortical interneurons," Nature 457(7233), 1137-1141 (2009).

89. R. Yuste and W. Denk, "Dendritic spines as basic functional units of neuronal integration," Nature 375(6533), 682-684 (1995).

90. J. Noguchi et al., "Spine-neck geometry determines NMDA receptordependent $\mathrm{Ca}^{2+}$ signaling in dendrites," Neuron 46(4), 609-622 (2005).

91. X. Chen et al., "Functional mapping of single spines in cortical neurons in vivo," Nature 475(7357), 501-505 (2011).

92. J. Schiller, Y. Schiller, and D. E. Clapham, "NMDA receptors amplify calcium influx into dendritic spines during associative pre- and postsynaptic activation," Nat. Neurosci. 1(2), 114-118 (1998).

93. B. L. Bloodgood, A. J. Giessel, and B. L. Sabatini, "Biphasic synaptic Ca influx arising from compartmentalized electrical signals in dendritic spines," PLoS Biol. 7(9), e1000190 (2009).

94. B. Scholl, D. E. Wilson, and D. Fitzpatrick, "Local order within global disorder: synaptic architecture of visual space," Neuron 96(5), 1127 1138.e4 (2017).

95. D. E. Wilson et al., "Orientation selectivity and the functional clustering of synaptic inputs in primary visual cortex," Nat. Neurosci. 19(8), 1003-1009 (2016)

96. M. F. Iacaruso, I. T. Gasler, and S. B. Hofer, "Synaptic organization of visual space in primary visual cortex," Nature 547(7664), 449-452 (2017).

97. M. Levy, A. E. Schramm, and P. Kara, "Strategies for mapping synaptic inputs on dendrites in vivo by combining two-photon microscopy, sharp intracellular recording, and pharmacology," Front. Neural Circuits 6, 101 (2012).

98. F. Gambino et al., "Sensory-evoked LTP driven by dendritic plateau potentials in vivo," Nature 515(7525), 116-119 (2014).

99. C. Grienberger, X. Chen, and A. Konnerth, "NMDA receptor-dependent multidendrite $\mathrm{Ca}^{2+}$ spikes required for hippocampal burst firing in vivo," Neuron 81(6), 1274-1281 (2014).
100. M. E. Larkum, J. J. Zhu, and B. Sakmann, "A new cellular mechanism for coupling inputs arriving at different cortical layers," Nature 398(6725), 338-341 (1999).

101. J. Waters and F. Helmchen, "Boosting of action potential backpropagation by neocortical network activity in vivo," J. Neurosci. 24(49), 11127-11136 (2004)

102. G. J. Stuart and M. Hausser, "Dendritic coincidence detection of EPSPs and action potentials," Nat. Neurosci. 4(1), 63-71 (2001).

103. M. E. Larkum, K. M. Kaiser, and B. Sakmann, "Calcium electrogenesis in distal apical dendrites of layer 5 pyramidal cells at a critical frequency of back-propagating action potentials," Proc. Natl. Acad. Sci. U. S. A. 96(25), 14600-14604 (1999).

104. C. Q. Chiu et al., "Compartmentalization of GABAergic inhibition by dendritic spines," Science 340(6133), 759-762 (2013).

105. M. Murayama and M. E. Larkum, "Enhanced dendritic activity in awake rats," Proc. Natl. Acad. Sci. U. S. A. 106(48), 20482-20486 (2009).

106. C. A. Baker et al., "Cellular resolution circuit mapping with temporalfocused excitation of soma-targeted channelrhodopsin," Elife 5, e14193 (2016).

107. G. J. Broussard et al., "In vivo measurement of afferent activity with axon-specific calcium imaging," Nat. Neurosci. 21(9), 1272-1280 (2018).

108. W. R. Zipfel, R. M. Williams, and W. W. Webb, "Nonlinear magic: multiphoton microscopy in the biosciences," Nat. Biotechnol. 21(11), 1369-1377 (2003).

109. M. E. Sheffield and D. A. Dombeck, "Calcium transient prevalence across the dendritic arbour predicts place field properties," Nature 517(7533), 200-204 (2015).

110. R. Lu et al., "Video-rate volumetric functional imaging of the brain at synaptic resolution," Nat. Neurosci. 20(4), 620-628 (2017).

111. H. Dana et al., "High-performance calcium sensors for imaging activity in neuronal populations and microcompartments," Nat. Methods 16, 649-657 (2019).

Farhan Ali is currently a postdoctoral fellow in the Kwan Lab, Department of Psychiatry, Yale University School of Medicine. His research employs in vivo imaging and physiological approaches to understand brain activity dynamics and processes such as learning and memory, and how these functions are disrupted in neuropsychiatric and neurodevelopmental disorders. He is also interested in translating neuroscience knowledge for applications in neuropsychiatry, psychology, and education. He received his BA degree in psychology from the National University of Singapore and a PhD in biology (neuroscience) from Harvard University.

Alex C. Kwan received his BASc degree in engineering physics from Simon Fraser University in 2003 and PhD in applied physics from Cornell University in 2009. He is currently an assistant professor in the Department of Psychiatry at Yale University. Research in his lab focuses on the role of the medial prefrontal cortex in decision-making and neuropsychiatric disorders. Many experiments involve developing and applying optical methods to record and control neural activity in behaving mice. 